\title{
Editorial: New Solutions for Smart Grids With High-Penetration Distributed Energy Resources
}

\author{
Liang Chen ${ }^{1}$, Chao Long ${ }^{2}$ and Yang $\mathrm{Li}^{3 *}$ \\ ${ }^{1}$ School of Automation, Nanjing University of Information Science and Technology, Nanjing, China, ${ }^{2}$ School of Water, Energy and \\ Environment, Cranfield University, Cranfield, United Kingdom, ${ }^{3}$ School of Electrical Engineering, Northeast Electric Power \\ University, Jilin, China
}

Keywords: distributed energy, smart grid, energy storage, cyber security, DC power system

Editorial on the Research Topic

New Solutions for Smart Grids With High-Penetration Distributed Energy Resources

OPEN ACCESS

Edited by:

S. M. Muyeen,

Qatar University, Qatar

Reviewed by:

Ioulia Papaioannou,

European Commission, Italy

*Correspondence:

Yang $\mathrm{LI}$

liyang@neepu.edu.cn

Specialty section:

This article was submitted to Smart Grids,

a section of the journal Frontiers in Energy Research

Received: 10 July 2021 Accepted: 17 December 2021

Published: 10 January 2022

Citation:

Chen L, Long C and Li Y (2022) Editorial: New Solutions for Smart

Grids With High-Penetration

Distributed Energy Resources.

Front. Energy Res. 9:739253.

doi: 10.3389/fenrg.2021.739253

\section{INTRODUCTION}

The application of distributed energy resources (DERs) is an effective way to solve the environmental pollution problem, and ensure a sustainable energy supply just like wind and solar energy. However, with the rapid developments of DERs, the high penetration of renewable energy will bring new challenges to power system operation due to the random fluctuation of power generation. Nowadays, new emerging techniques in smart grid, such as power storages, cyber-physical systems and DC transmission systems, provide more regulatory methods for enabling higher penetration of DERs.

This special issue is organized to introduce the latest research in the field of high penetration of DERs. At present, 18 papers have been accepted for this special issue. These papers can be sorted into the following 7 categories including: 1) Cyber security; 2) DC transmission system;3) Energy storage; 4) Short-circuit current calculation of wind turbines; 5) Energy trading; 6) Parameter and topology identification; 7) other topics. The sections below introduce the major topics of the papers covered in each category.

\section{CYBER SECURITY TECHNIQUES}

The security and economic operation of power system with high penetration of DERs relies on the accurate operating information which can be obtained by measuring equipment and communication systems. However, with the rapid developments of information technologies, the cyber-attacks on smart grids cause blackouts and disrupt power systems. Aiming at this problem, the detection methods of cyber-attacks have been developed to ensure that the accurate information can be obtained. Qu et al. propose a novel detection method of the false data injection attack based on cyberphysical genes. Wang et al. propose a model for cyber and physical data fusion using data link 
technologies for detecting attacks on a cyber-physical power system. Wang et al. propose a reconstruction method for missing data in power system measurements based on least squares generative adversarial networks, which is able to reconstruct the missing data in the case of high latitude and high loss rate.

\section{FAULT ANALYSIS AND CONTROL OF DC TRANSMISSION SYSTEMS}

High-voltage direct current (HVDC) transmission has already been applied in power systems. The HVDC transmission is more flexible than AC transmission system, which can enhance the penetrations of DERs. On the other hand, the DC fault will seriously affect the operation safety of power systems. Sun et al. establish the mathematical model of modular multilevel converter considering $\mathrm{AC}$ feed, and an improved analysis method of single-pole grounding fault of flexible DC network grounded by metal loop is proposed. Li et al. propose a region boundaries determination method for critical commutation failures in multi-infeed HVDC systems under unbalanced short circuit faults. Mao et al. propose a rapid recovery strategy of hybrid Multi-infeed HVDC after AC failure, which can effectively suppress continuous commutation failure and improve the recovery speed of $\mathrm{AC}$ voltage and DC power.

\section{ENERGY STORAGE IN SMART GRIDS}

Large scale penetration of renewable energy is a challenge for power systems due to the reason that the AC power networks are lack of sufficient flexibilities to deal with the random fluctuations of power sources. Energy storage is an effective way to deal with the penetration problem of DERs. Yang et al. decouple the insufficient flexibility and wind power curtailment caused by transmission congestion, and then a joint planning model of energy storage system and transmission congestion is established. Li et al. establish an optimization method of energy storage system (ESS) to optimize the capacities and locations of distributed ESS to support the voltage regulation of distribution networks.

\section{SHORT-CIRCUIT CURRENT CALCULATION OF WIND TURBINES}

Doubly-fed induction generators (DFIGs) are one of the most common kinds of wind generators used in power systems. It is necessary to analyze the influences of short-circuit currents on a power system. Yin proposed a calculation method of the shortcircuit current of DFIGs considering the influence of rotor current dynamics. The author also studies the short-circuit current calculation method of a DFIG at the initial fault time with uninterrupted excitation based on the characteristics of non-abrupt changes of flux linkage.

\section{ELECTRICITY PRICE AND TRADING}

The demand response is an effective way to enhance the power system flexibility and promote the penetration of DERs. The electricity price is a main regulation method of power loads, which can respond to the power balance of smart grid. Li et al. study a real-time price decision model which adjusts the price in an acceptable range of heating users. Li et al. review the recent studies and projects of energy trading based on blockchain.

\section{PARAMETER AND TOPOLOGY IDENTIFICATION}

The random fluctuations of DERs make power system dynamics more frequent than before. The accurate parameters and topology of power grids are vital for the control and management of power dynamics. Qu et al. propose a dynamic optimization method of transmission line parameters based on grey support vector regression. Wang et al. propose a method to identify the topology of a power network based on a knowledge graph and the graph neural network, which can adapt to errors and informational conflicts in the graph data.

\section{OTHER TOPICS}

Aiming at the allocation problem of service resources, Jin et al. propose a data association fusion method based on an improved simhash algorithm for allocating the address of power customers according to the short text and unstructured characteristics of the address. Huang et al. propose a calculation method for the temperature rise of oil-immersed transformers considering the vibration of insulating oil. Fu et al. analyze the driving forces of $\mathrm{CO}_{2}$ emissions change of manufacturing industry of China, and conclude that it is necessary to develop a smart grid to reduce the proportion of coal in the energy structure and increase the proportion of renewable energy. Zhang et al. investigate the economic feasibility of grid-balancing plants via a systematic overall decomposition-based methodology for real geographical zones and flexibility-need scenarios.

\section{CONCLUSION}

The papers in this special issue cover different techniques in the smart grid with high penetration of DERs. The outcomes of these research works indicate that the emerging techniques are essential and helpful to promote the penetration of DERs.

\section{AUTHOR CONTRIBUTIONS}

All authors listed have made a substantial, direct and intellectual contribution to the work, and approved it for publication. 


\section{FUNDING}

This work is partly supported by the Natural Science Foundation of Jilin Province, China under Grant No. YDZJ202101ZYTS149.

Conflict of Interest: The authors declare that the research was conducted in the absence of any commercial or financial relationships that could be construed as a potential conflict of interest.

Publisher's Note: All claims expressed in this article are solely those of the authors and do not necessarily represent those of their affiliated organizations, or those of the publisher, the editors and the reviewers. Any product that may be evaluated in this article, or claim that may be made by its manufacturer, is not guaranteed or endorsed by the publisher.

Copyright $\odot 2022$ Chen, Long and Li. This is an open-access article distributed under the terms of the Creative Commons Attribution License (CC BY). The use, distribution or reproduction in other forums is permitted, provided the original author(s) and the copyright owner(s) are credited and that the original publication in this journal is cited, in accordance with accepted academic practice. No use, distribution or reproduction is permitted which does not comply with these terms. 